\title{
Morbimortality assessment in abdominal surgery: are we predicting or overreacting?
}

\author{
Sebastian Valenzuela', Laura Niño², Danny Conde ${ }^{1,2^{*}}$ (D) Felipe Girón ${ }^{1,2,3}$ (D) , Lina Rodríguez ${ }^{3}$ (D)

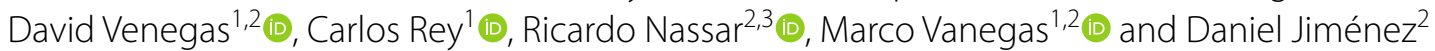

\begin{abstract}
Background: High-risk surgical procedures represent a fundamental part of general surgery practice due to its significant rates of morbidity and mortality. Different predictive tools have been created in order to quantify perioperative morbidity and mortality risk. POSSUM (Physiological and Operative Severity Score for the enumeration of Mortality and morbidity) is one of the most widely validated predictive scores considering physiological and operative variables to precisely define morbimortality risk. Nevertheless, seeking greater accuracy in predictions P-POSSUM was proposed. We aimed to compare POSSUM and P-POSSUM for patients undergoing abdominal surgery.
\end{abstract}

Methods: A retrospective observational study with a prospective database was conducted. Patients over 18 years old who complied with inclusion criteria between 2015 and 2016 were included. Variables included in the POSSUM and P-POSSUM Scores were analyzed. Descriptive statistics of all study parameters were provided. The analysis included socio-demographic data, laboratory values, and imaging. Bivariate analysis was performed.

Results: 350 Patients were included in the analysis, $55.1 \%$ were female. The mean age was $55.9 \pm 20.4$ years old. POSSUM revealed a moderated index score in $61.7 \%$ of the patients, mean score of 12.85 points $\pm 5.61 .89 .1 \%$ of patients had no neoplastic diagnosis associated. Overall morbidity and mortality rate was $14.2 \%$ and $7.1 \%$. P-POSSUM could predict more precisely mortality $(p<0.00)$.

Conclusions: The POSSUM score is likely to overestimate the risk of morbidity and mortality in patients with high/ moderate risk, while the P-POSSUM score seems to be a more accurate predictor of mortality risk. Further studies are needed to confirm our results.

Keywords: Risk prediction model, Mortality, Critical care, Laparotomy, Prognosis

\section{Background}

High-risk surgical procedures still carry considerable risk for patients in spite of advances in surgical technique and perioperative care thus making it of paramount importance the development of tools that predict postoperative outcomes for patient populations in specific surgical settings [1]. The optimal methods describe individual patient risk and account for a surgical procedure allowing

\footnotetext{
*Correspondence: condedanny889@gmail.com

${ }^{1}$ Department of Surgery, Hospital Universitario Mederi, Calle 103a 21-93,

110111 Bogotá, D.C, Colombia

Full list of author information is available at the end of the article
}

preoperative optimization in individual postoperative settings [1]. This may lead to improve not only the decision-making process during the hospital stay, but also, allows clearer and more precise communication with patients and families in terms of expectations and possible outcomes [1]. Therefore, considering the growing need for better operative and medical decisions based on early identification of patients' high risk of morbimortality, multiple risk scores have emerged ranging from preoperative to postoperative scales [2].

Numerous studies have assessed its accuracy to predict life-threatening and non-life-threatening

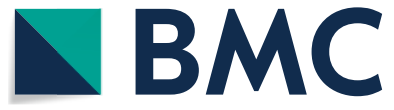

(c) The Author(s) 2022. Open Access This article is licensed under a Creative Commons Attribution 4.0 International License, which permits use, sharing, adaptation, distribution and reproduction in any medium or format, as long as you give appropriate credit to the original author(s) and the source, provide a link to the Creative Commons licence, and indicate if changes were made. The images or other third party material in this article are included in the article's Creative Commons licence, unless indicated otherwise in a credit line to the material. If material is not included in the article's Creative Commons licence and your intended use is not permitted by statutory regulation or exceeds the permitted use, you will need to obtain permission directly from the copyright holder. To view a copy of this licence, visit http://creativecommons.org/licenses/by/4.0/. The Creative Commons Public Domain Dedication waiver (http://creativeco mmons.org/publicdomain/zero/1.0/) applies to the data made available in this article, unless otherwise stated in a credit line to the data. 
complications (defined by Clavien-Dindo classification 4-5) finding associations with significant statistical value. [2-4]. Copeland et al. developed the POSSUM (Physiological and Operative Severity Score for the enumeration of Mortality and morbidity) scoring system to help provide both retrospective and prospective analysis of the risk of postsurgical mortality and morbidity. The POSSUM score describes 18 factors in two component parts: 12 physiological factors (PS) and 6 operative factors (OS). From these values predicted mortality can be calculated [5] as it was developed as a tool to compare morbidity and mortality in a wide range of general surgical procedures to compare the performance of individual surgical units [1].

Given that the POSSUM scoring system was found to consistently overestimate the mortality rate in lowrisk patients [6], in 1998 the need to adjust logistic regression analysis used in POSSUM scoring to better predict mortality resulted in a second scoring system using the same standard data, Portsmouth-POSSUM (P-POSSUM). This scoring system collects the same 18 physiological and operative parameters with a different calculation formula to predict mortality [7]. There on, both systems are used today helping guide utilization of health care resources for postoperative patients [8].

In terms of mortality, both Raut et al. and Mohammed et al. proved the accuracy of POSSUM and P-POSSUM scores in predicting mortality risk, with more than $80 \%$ of accuracy in selected patients $[9,10]$. Additionally, Prabakaran et al. found that selected colorectal surgery patients have an increased prediction force, for that reason a subclass of POSSUM (CR-POSSUM) has been described in these populations [11]. Nevertheless, it has been described that score results shouldn't be generalized to all patients that underwent surgical procedures and have to be personalized and analyzed according to each population [12].

Although good surgical technique is paramount in reducing adverse outcomes, these are dependent on the physiological state of patients, operative severity, and perioperative support devices [13]. The need for early identification of patients who are at high risk of mortality is present to outline and improve surgical outcomes and postoperative care [14]. The capability to predict life-threatening and high-cost complications may lead to an impact on the quality of life, and mortality rates in surgical patients. Hence, our aim is to describe the experience using POSSUM and P-POSSUM risk scores to predict morbidity and mortality in patients that underwent urgent abdominal surgical procedures in a high complexity hospital.

\section{Methods}

Following Institutional Review Board approval, and after calculating a sample size of 350 using Dobson formula with a 95\% confidence level. Ethical compliance with the Helsinki Declaration, current legislation on research Res. 008,430-1993 and Res. 2378-2008 (Colombia) and the International Committee of Medical Journal Editors (ICMJE) were ensured under our Ethics and Research Institutional Committee (IRB) approval.

\section{Patient's description}

The study includes all patients over 18 years old who underwent abdominal surgical procedures between 2015 and 2016 in a single institution. We exclude patients referred to our center with extra-institutional surgical management and those with missing data in the medical description. Morbidity was assessed by the clinical record, imagenologic findings, and surgical data, and classified according to the international Clavien-Dindo classification. Malignant disease was confirmed by histopathological analysis after surgery.

Data included patients' demographics, and all clinical, imaging, and blood chemistry needed for calculating POSSUM and P-POSSUM scores. Descriptive statistics of all study parameters were provided. Data were analyzed using IBM SPSS Statistics 25 software. Continuous data were summarized by their mean, standard deviation, median, minimum and maximum. Categorical data were summarized by their frequency and proportion. Bivariate analysis was performed. Qualitative variables were analyzed using chi-square statistics (Fisher's exact test when appropriate). Quantitative variables were analyzed, based on normality, with Spearman's or Pearson's association's correlation coefficient accordingly. Bivariate analysis between qualitative and quantitative variables was performed using the Mann-Whitney test or the t-test for independent samples.

\section{Results}

350 patients underwent urgent abdominal procedures, they were predominantly female $(55.4 \%)$ with a mean age of 55.91 years old (Table 1). Among the clinical parameters measured, the mean systolic blood pressure was

Table 1 Demographic characteristics

\begin{tabular}{ll}
\hline Variable & Value \\
\hline Sex & \\
$\quad$ Male & $156(44.5 \%)$ \\
Female & $194(55.4 \%)$ \\
Age (years) (mean \pm SD) & $55.91 \pm 20.4$ \\
\hline
\end{tabular}


$125 \mathrm{mmHg}$ and the average heart rate was 85.8 beats per minute, most of the patients did not have dyspnea $(89.4 \%)$ or present signs or symptoms of heart failure (59.4\%). However, an important group of patients (35.7\%) received cardiovascular pharmacological management.
In most cases, the Glasgow Score was not suggestive of head injury (14.9 \pm 0.734$)$ (Table 2).

Within blood chemistry, mean leukocyte count was $11,818 \mathrm{uL} \pm 5324$, mean hemoglobin of $13.97 \mathrm{~g} /$ $\mathrm{dl} \pm 4.15$, normal renal function with a BUN of $17.54 \mathrm{mg} /$

Table 2 Paraclinical and clinical characteristics

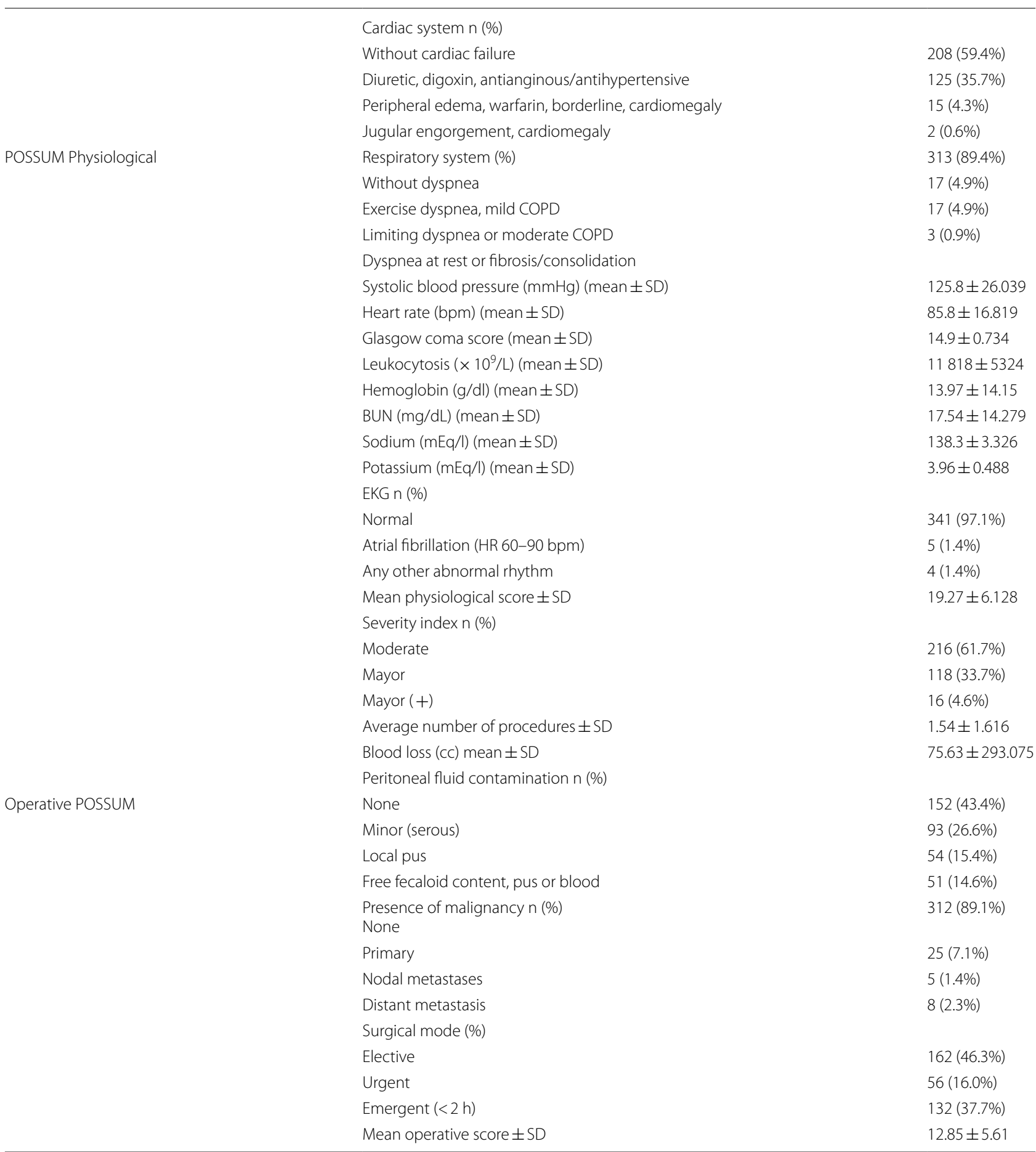


Table 3 Morbidity and mortality

\begin{tabular}{ll}
\hline Morbidity $\mathbf{n}(\%)$ & \\
\hline Clavien-Dindo I & $2(0.6 \%)$ \\
Clavien-Dindo II & $16(4.6 \%)$ \\
Clavien-Dindo IIIa & $7(2.0 \%)$ \\
Clavien-Dindo IIIb & $23(6.6 \%)$ \\
Clavien-Dindo IVa & $1(0.3 \%)$ \\
Clavien-Dindo IVb & $1(0.3 \%)$ \\
Mortality n (\%) & $25(7.1 \%)$ \\
\hline
\end{tabular}

Table 4 Pearson's correlation

\begin{tabular}{lll}
\hline \% Mortality & \% Mortality & P-POSSUM \\
\hline Pearson Correlation & 1 & 0.948 \\
$\mathbf{p}$ & 350 & 0.000 \\
$\mathbf{n}$ & & 349 \\
\hline
\end{tabular}

$\mathrm{dl} \pm 14.279$, electrolytes with sodium $138.3 \mathrm{mEq} / \mathrm{l} \pm 3.326$ and potassium $3.96 \mathrm{mEq} \pm 0.488$, both within normal limits. Admission electrocardiogram was normal in 97.1\% of patients. Mean physiological POSSUM was $19.27 \pm 6.113$ points.

On the other hand, the mean operative POSSUM severity index was $12.85 \pm 5.61$ (moderate). $46.3 \%$ of patients underwent elective surgery, $16 \%$ urgent and $37.7 \%$ emergent. Mean blood loss was 75.63 cc. Peritoneal fluid had some change in appearance in half the patients, $26.6 \%$ was serous fluid, $15.4 \%$ had local pus, and $14.6 \%$ had free fecaloid content, pus, or blood. $89.1 \%$ did not present neoplasms, the remaining $10 \%$ had evidence of primary neoplasia (7.1\%), nodal metastases (1.4\%), or distant metastatic disease (2.3\%). Mean operative POSSUM was 12.85 points \pm 5.653 .

Morbidity was analyzed using the Clavien-Dindo classification, 50 patients (14.2\%) had some type of morbidity. Clavien-Dindo I was present in 2 patients that required hydro electrolytic replacement. In the case of Clavien-Dindo II, 10 patients required blood transfusions and the rest one's parenteral nutrition. Clavien-Dindo IIIa was explained by patients that need percutaneous drainage of intra-abdominal collections, and type IIIb the remaining patients that need surgical intervention due to abdominal complications that conservative management fails. Clavien-Dindo IV was explained by patients who present respiratory failure and require orotracheal intubation. $7.1 \%$ mortality rate was documented (ClavienDindo V) (Table 3). Finally, we found a strong association between the P-POSSUM score and mortality (Pearson Coeff. $0.948 \mathrm{p}=0.000)($ Table 4$)$.

\section{Discussion}

Surgical risk assessment should be performed by a surgical team seeking a clear perspective not only for possible clinical outcomes but also in order to give patients and families a clear standpoint in terms of expectations [2]. Raw mortality and morbidity, though commonly reported, can be misleading due to differences in preoperative and intraoperative findings of patients $[6,15]$. Although the good surgical technique is paramount in reducing adverse outcomes, the final result is a compound where the physiological state of the patient, operative severity, and peri-operative support services intervene [7]. Patient evolution and surgical complications depend on several factors: the quality of the surgical team, physiological status, and surgical stress among others [5]. Even though POSSUM and P-POSSUM scales have been widely used in Europe, their spread through America has not been equal [4].

The P-POSSUM scale was created in order to correct the elevated mortality risk obtained by POSSUM [3]; some studies showed an increased accuracy of P-POSSUM over POSSUM in mortality prediction, with near results with the observed mortality $[9-12,16]$. Given that P-POSSUM has to be correlated to the general condition of the local population for it to be effective [9-13], variability of results obtained is explained by lack of adjustments to population baseline characteristics that generate changes in predicted values. Among these characteristics are malnutrition and economic status; nevertheless literature, as our study, reports P-POSSUM scale has an increased accuracy to predict mortality compared with POSSUM in emergency surgery [12].

We assessed risk prediction in terms of morbidity and mortality by POSSUM and P-POSSUM scores. $61.7 \%$ of the patients underwent emergent surgical procedures with a moderate operative score, suggesting an increased risk of morbidity in the first 30 days after surgery. Nonetheless, actual morbidity in our population was significantly lower than predicted (14.2\%), most of them classified as Clavien-Dindo II (4.6\%) and IIIB (6.6\%) were $46.6 \%$ of patients required a second surgical procedure, matching Gonzalez-Martinez et al. findings which sustained mortality and morbidity values calculated by POSSUM are overestimated $[1,17]$. Stonelake et al. described 86 patients who underwent urgent laparotomy with a $77.9 \%$ of morbidity $[2,14]$, results higher than those found in our population (14.2\%). Nonetheless, in terms of mortality, in our study, a higher mortality rate was documented compared to Stonelake et al. results $(7.1 \%$ vs $5.81 \%)[2,14]$. This could be due to the not only surgery findings but also to the previous clinical status of the patients involved in our study, reflected in their higher physiological and surgical values. 
Melendez et al., analyzed 513 patients for risk prediction using the POSSUM scale finding overestimated morbidity and mortality rates compared with actual values of $17.56 \%$ vs $10.33 \%$ and $4.5 \%$ vs $1.75 \%$ respectively [3, 18]. Additionally, P-POSSUM was more accurate, with a predicted mortality value of $1.6 \%$ vs an observed value of $1.75 \%$ [3, 18]. Mortality and morbidity outcomes in the Melendez cohort showed that the overestimation persisted to a lesser extent, which relates to our findings where values of mortality calculated by POSSUM score overestimated mortality rates and P-POSSUM score estimations had results closer to real (mortality rate $7.1 \% \mathrm{Vs}$ P-POSSUM predicted mortality 6.31\%), with a Pearson's coefficient 0.948 showing clear statistical correlation $(\mathrm{p}<0.000)$. Additionally, most of the studies find discrepancies between expected and observed mortality with the use of POSSUM, but similar with P-POSSUM [1].

Despite the effectiveness of POSSUM and P-POSSUM scores, improvements are still needed because of the overinterpretation in morbidity and mortality, specifically in high complexity surgeries [8, 19-22]. Among the limitations of this study is its retrospective nature, data was obtained from previously collected clinical charts which may result in missing variables for some of the observations. Thus, more studies are needed to confirm our results.

\section{Conclusions}

Overall, POSSUM seems to overestimate both morbidity and mortality. Whereas P-POSSUM appears to be closer to reality, it may be more accurate in estimating mortality in patients with moderate to high risk; adjustment to baseline characteristics of the population could increase the accuracy. Even though P-POSSUM seems like a feasible score to predict morbimortality in abdominal surgery, further studies are needed to confirm our results.

\section{Abbreviations}

POSSUM: Physiological and Operative Severity Score for the enumeration of Mortality and morbidity; P-POSSUM: Portsmouth-Physiological and Operative Severity Score for the enumeration of Mortality and morbidity; PS: Physiological factors; OS: Operative factors; COPD: Chronic obstructive pulmonary disease.

\section{Acknowledgements}

To our institution and to our patients.

\section{Authors' contributions}

All authors contributed to the study conception and design. Material preparation, data collection, and analysis were performed by SV, DJ, and LN. The first draft of the manuscript was written by DC, FG, LR, DV, CR, and MV, and all authors commented on previous versions of the manuscript. All authors read and approved the final manuscript.

\section{Funding}

This research did not receive any specific grant from funding agencies in the public, commercial, or not-for-profit sectors.

\section{Availability of data and materials}

Dataset and materials used during the current study are available from the corresponding author on request.

\section{Declarations}

Ethics approval and consent to participate

Ethical compliance with the Helsinki Declaration, current legislation on research Res. 008430-1993 and Res. 2378-2008 (Colombia) and the International Committee of Medical Journal Editors (ICMJE) were ensured under our institutional Ethics and Research Institutional Committee (IRB) approval. Written informed consent was obtained from all participants for the execution of this study.

\section{Consent for publication}

Not applicable.

\section{Competing interests}

All authors certify that they have no affiliations with or involvement in any organization or entity with any financial interest or non-financial interest in the subject matter or materials discussed in this manuscript.

\section{Author details}

${ }^{1}$ Department of Surgery, Hospital Universitario Mederi, Calle 103a 21-93, 110111 Bogotá, D.C, Colombia. ${ }^{2}$ School of Medicine, Universidad del Rosario, Bogotá, Colombia. ${ }^{3}$ School of Medicine, Universidad de los Andes, 111711 Bogotá, Colombia.

Received: 14 September 2021 Accepted: 24 December 2021 Published online: 18 January 2022

References

1. Scott S, Lund JN, Gold S, Elliott R, Vater M, Chakrabarty MP, Heinink TP, Williams JP. An evaluation of POSSUM and P-POSSUM scoring in predicting post-operative mortality in a level 1 critical care setting. BMC Anesthesiol. 2014;18(14):104. https://doi.org/10.1186/1471-2253-14-104.

2. Stonelake $S$, Thomson $P$, Suggett N. Identification of the high risk emergency surgical patient: which risk prediction model should be used? Ann Med Surg (Lond). 2015;4(3):240-7. https://doi.org/10.1016/j.amsu.2015. 07.004

3. Jones DR, Copeland GP, de Cossart L. Comparison of POSSUM with APACHE II for prediction of outcome from a surgical high-dependency unit. Br J Surg. 1992;79(12):1293-6. https://doi.org/10.1002/bjs.18007 91216.

4. Kumar P, Rodrigues GS. Comparison of POSSUM and P-POSSUM for risk-adjusted audit of patients undergoing emergency laparotomy. Ulus Travma Acil Cerrahi Derg. 2009;15(1):19-22.

5. Copeland GP, Jones D, Walters M. POSSUM: a scoring system for surgical audit. Br J Surg. 1991;78(3):355-60. https://doi.org/10.1002/bjs.18007 80327.

6. Whiteley MS, Prytherch DR, Higgins B, Weaver PC, Prout WG. An evaluation of the POSSUM surgical scoring system. Br J Surg. 1996;83(6):812-5. https://doi.org/10.1002/bjs.1800830628.

7. Prytherch DR, Whiteley MS, Higgins B, Weaver PC, Prout WG, Powell SJ. POSSUM and Portsmouth POSSUM for predicting mortality. Physiological and Operative Severity Score for the enUmeration of Mortality and morbidity. Br J Surg. 1998;85(9):1217-20. https://doi.org/10.1046/j.1365-2168. 1998.00840.x

8. Scott S, Lund JN, Gold S, Elliott R, Vater M, Chakrabarty MP, Heinink TP, Williams JP. An evaluation of POSSUM and P-POSSUM scoring in predicting post-operative mortality in a level 1 critical care setting. BMC Anesthesiol. 2014;14:104. https://doi.org/10.1186/1471-2253-14-104.

9. Raut NR, Maibam C, Singh J, Devi SR, Singh TAK. Portsmouth physiological and operative severity score for the enumeration of mortality and morbidity scoring system in general surgical practice and identifying risk factors for low outcome. J Media Sociol. 2013:27:119-23.

10. Mohammed S. Comparison between POSSUM and P-POSSUM scores in prediction of post-operative mortality and morbidity in patients 
undergoing emergency laparotomy at Omdurman teaching hospital, Scholars. J Appl Med Sci. 2015;3:650-3.

11. Prabakaran V, Thangaraju T, Mathew AC, Govindan V, Kannan V, Poulose TR. CR-POSSUM-can it be used to predict morbidity? A single-centre retrospective study. Indian J Surg Oncol. 2019;10(1):174-9. https://doi. org/10.1007/s13193-018-0841-8.

12. Nichols KG, Prytherch DR, Fancourt MF, Gilkison WT, Kyle SM, Mosquera DA. Risk-adjusted general surgical audit in octogenarians. ANZ J Surg. 2008:78(11):990-4. https://doi.org/10.1111/j.1445-2197.2008.04718.x.

13. Ghaferi AA, Birkmeyer JD, Dimick JB. Variation in hospital mortality associated with inpatient surgery. N Engl J Med. 2009;361 (14):1368-75. https:// doi.org/10.1056/NEJMsa0903048.

14. Stonelake S, Thomson P, Suggett N. Identification of the high risk emergency surgical patient: which risk prediction model should be used? Ann Med Surg (Lond). 2015;4(3):240-7. https://doi.org/10.1016/j.amsu.2015. 07.004 .

15. Ngulube A, Muguti GI, Muguti EG. Validation of POSSUM, P-POSSUM and the surgical risk scale in major general surgical operations in Harare: a prospective observational study. Ann Med Surg (Lond). 2019;41:33-9. https://doi.org/10.1016/j.amsu.2019.03.007.

16. Wang $H$, Wang $H$, Chen T, Liang $X$, Song $Y$, Wang J. Evaluation of the POSSUM, P-POSSUM and E-PASS scores in the surgical treatment of hilar cholangiocarcinoma. World J Surg Oncol. 2014;12:191. https://doi.org/10. 1186/1477-7819-12-191.

17. González-Martínez S, Martín-Baranera M, Martí-Saurí I, Borrell-Grau N, Pueyo-Zurdo JM. Comparison of the risk prediction systems POSSUM and P-POSSUM with the Surgical Risk Scale: A prospective cohort study of 721 patients. Int J Surg. 29:19-24. https://doi.org/10.1016/j.ijsu.2016.03.005.

18. Meléndez HJ, Contreras JR. Validación de los índices POSSUM y Portsmouth-POSSUM en cirugía general en dos instituciones de segundo nivel. Revista Colombiana de Anestesiología. 2008;36(4):249-57. https:// doi.org/10.1016/50120-3347(08)64002-2.

19. Chen T, Wang H, Wang H, Song Y, Li X, Wang J. POSSUM and P-POSSUM as predictors of postoperative morbidity and mortality in patients undergoing hepato-biliary-pancreatic surgery: a meta-analysis. Ann Surg Oncol, 2013;20(8):2501-10. https://doi.org/10.1245/s10434-013-2893-x.

20. Stonelake S, Thomson P, Suggett N. Identification of the high risk emergency surgical patient: Which risk prediction model should be used? Annals Med Surg. 2015;4(3) 240-247. https://doi.org/10.1016/j.amsu.2015. 07.004 .

21. Sagar PM, Hartley MN, MacFie J, Taylor BA, Copeland GP. Comparison of individual surgeon's performance. Risk-adjusted analysis with POSSUM scoring system. Dis Colon Rectum. 1996;39(6):654-8. https://doi.org/10. 1007/BF02056945.

22. Echara ML, Singh A, Sharma G. Risk-Adjusted Analysis of Patients Undergoing Emergency Laparotomy Using POSSUM and P-POSSUM Score: A Prospective Study. Niger J Surg. 2019;25(1):45-51. https://doi.org/10. 4103/njs.NJS_11_18.

\section{Publisher's Note}

Springer Nature remains neutral with regard to jurisdictional claims in published maps and institutional affiliations.

Ready to submit your research? Choose BMC and benefit from:

- fast, convenient online submission

- thorough peer review by experienced researchers in your field

- rapid publication on acceptance

- support for research data, including large and complex data types

- gold Open Access which fosters wider collaboration and increased citations

- maximum visibility for your research: over $100 \mathrm{M}$ website views per year

At BMC, research is always in progress.

Learn more biomedcentral.com/submissions 